\title{
The reproducibility and responsiveness of the lung clearance index in bronchiectasis
}

\author{
Lizzie Grillo ${ }^{1,2}$, Samantha Irving ${ }^{3}$, David M. Hansell ${ }^{4,5}$, Arjun Nair $^{4}$, \\ Bertrand Annan 4 , Simon Ward ${ }^{6}$, Diana Bilton ${ }^{5,7}$, Eleanor Main ${ }^{2}$, Jane Davies ${ }^{3,5}$, \\ Andrew Bush ${ }^{3,5}$, Robert Wilson ${ }^{5,7}$ and Michael R. Loebinger ${ }^{5,7}$ \\ Affiliations: ${ }^{1}$ Physiotherapy Department, Royal Brompton Hospital, London, UK. ${ }^{2}$ Institute for Child Health, \\ University College, London, UK. ${ }^{3}$ Department of Paediatrics, Royal Brompton Hospital, London, UK. \\ ${ }^{4}$ Department of Radiology, Royal Brompton Hospital, London, UK. ${ }^{5}$ Imperial College, London, UK. ${ }^{6}$ Department \\ of Physiology, Royal Brompton Hospital, London, UK. ${ }^{7}$ Host Defence Unit, Royal Brompton Hospital, London, UK.
}

Correspondence: Michael R Loebinger, Royal Brompton Hospital, London, SW3 6NP.

E-mail: m.loebingerarbht.nhs.uk

ABSTRACT Lung clearance index (LCI) is a potential clinical outcome marker in bronchiectasis. Its responsiveness to therapeutic intervention has not been determined. This study evaluates its responsiveness to a session of physiotherapy and intravenous antibiotic treatment of an exacerbation.

32 stable and 32 exacerbating bronchiectasis patients and 26 healthy controls were recruited. Patients had LCI and lung function performed before and after physiotherapy on two separate occasions in the stable patients and at the beginning and end of an intravenous antibiotic course in the exacerbating patients.

LCI was reproducible between visits in 25 stable patients, with an intraclass correlation of 0.978 (0.948, $0.991 ; \mathrm{p}<0.001)$. There was no significant difference in LCI (mean \pm SD) between stable $11.91 \pm 3.39$ and exacerbating patients $12.76 \pm 3.47$, but LCI was significantly higher in both bronchiectasis groups compared with healthy controls $(7.36 \pm 0.99) \quad(\mathrm{p}<0.001)$. Forced expiratory volume in $1 \mathrm{~s}$ improved after physiotherapy, as did alveolar volume after intravenous antibiotics, but LCI did not change significantly.

LCI is reproducible in stable bronchiectasis but unlike conventional lung function tests, is unresponsive to two short-term interventions and hence is unlikely to be a useful clinical tool for short-term acute assessment in these patients. Further evaluation is required to establish its role in milder disease and in the evaluation of long-term interventions.

@ERSpublications

LCI is unresponsive to airway clearance or i.v. antibiotic treatment of a pulmonary exacerbation in bronchiectasis http://ow.ly/PHI2a

Received: Jan 272015 | Accepted after revision: June 192015 | First published online: Sept 042015

This article has supplementary material available from erj.ersjournals.com

Support statement: Bayer Pharmaceuticals provided financial support towards the cost of this project but were not involved with the study design, patient recruitment, data collection or analysis. They have not contributed to the final write up or review of this manuscript. This study was supported by the NIHR Respiratory Disease Biomedical Research Unit at the Royal Brompton and Harefield NHS Foundation Trust and Imperial College London.

Conflict of interest: Disclosures can be found alongside the online version of this article at erj.ersjournals.com

Copyright @ERS 2015 


\section{Introduction}

Bronchiectasis is a chronic, progressive condition characterised by destruction of the bronchial wall and airway dilation as demonstrated on high resolution computerized tomography (HRCT) [1]. There are few evidence-based treatments available with several recent trials having mixed or inconclusive results [2]. Clinical management and trial design are limited by the lack of relevant clinical outcome measures [3]. Whereas forced expired volume in $1 \mathrm{~s}(\mathrm{FEV} 1)$ has been successfully utilised in cystic fibrosis (CF), it is a much less useful marker in other causes of bronchiectasis with a lack of clinically important change in multiple studies $[4,5]$.

The lung clearance index (LCI) is a measure of ventilation inhomogeneity in the peripheral airways. It is a reliable and valid test in both healthy and diseased adults and children [6-10]. In CF, the LCI has been shown to be more sensitive in evaluating early damage than other physiological measurements [6], including FEV1. Preliminary evidence suggests this may also be true in bronchiectasis. A recent study demonstrated that LCI was a reproducible marker in patients with bronchiectasis with the LCI score better correlated to CT scan abnormalities than other spirometry measures [11]. For this marker to make a significant impact in bronchiectasis management and trial design, it also needs to demonstrate responsiveness to changes in clinical condition of patients.

There have been few studies using LCI to assess responsiveness to treatment in CF, but none in other causes of bronchiectasis, where clinical endpoints are desperately needed. In CF, a treatment effect has been demonstrated with dornase alpha [12], hypertonic saline [13] and post treatment for a pulmonary exacerbation [14-16], although the clinically meaningful change in LCI has not yet been determined. In contrast, short-term physiotherapy was not found to have a consistent effect on the LCI results in a cohort of stable CF patients [17]. We hypothesised that LCI would be a more sensitive measure of treatment benefit than standard lung function tests in bronchiectasis. We measured LCI and standard pulmonary function tests before and after a session of airway clearance in stable and exacerbating patients, and before and after a course of intravenous antibiotic treatment of a pulmonary exacerbation.

\section{Patients and methods}

We prospectively recruited healthy controls, patients with stable bronchiectasis (no antibiotics in the previous 4 weeks) and patients admitted with an exacerbation of bronchiectasis (figure 1a and b). Bronchiectasis was confirmed by review of HRCT performed within 6 months of the study. Exacerbation was defined as at least two of increased cough, sputum volume or purulence requiring hospital admission and intravenous antibiotics as determined by the admitting consultant. Approval for the study was given by the National Research Ethics Service, South East Coast NRES Committee (RECreference: 12/LO/0345).

Stable bronchiectasis participants attended two identical visits no more than a week apart. Assessments included LCI in triplicate followed by pulmonary function tests (PFTs; comprising spirometry, plethysmographic lung volumes and carbon monoxide transfer). PFTs were converted to Z (SD) scores. Assessments were completed before (pre) and after (post) an airway clearance session by a physiotherapist.

a)

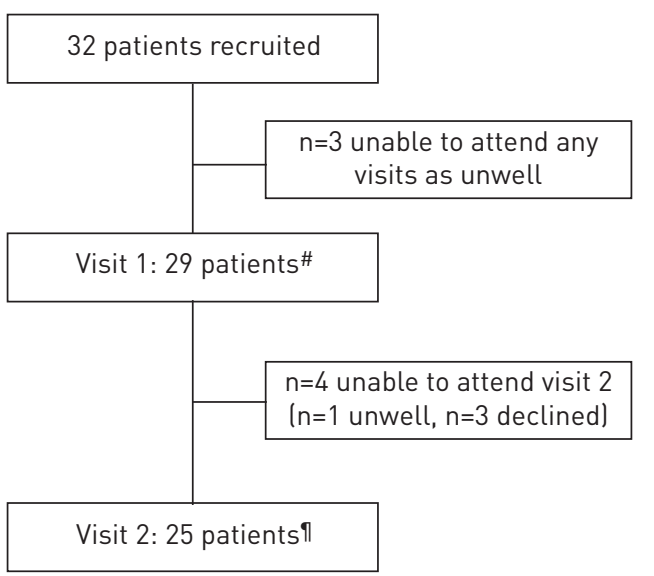

b)

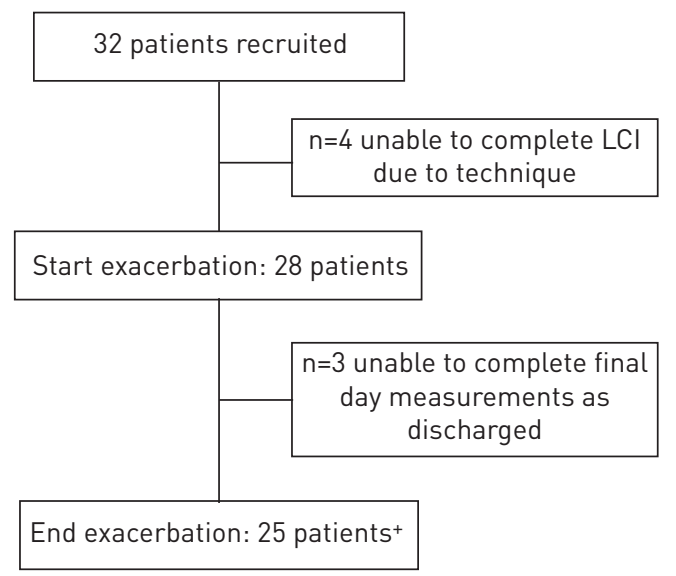

FIGURE 1 a) Consort diagram for the stable bronchiectasis group; and b) consort diagram for the exacerbation bronchiectasis group. ${ }^{\#}: \mathrm{n}=4$ unable to complete post-physiotherapy lung clearance index (LCI) visit 1 data ( $=2$ leaks, $n=2$ measurement error); ${ }^{\eta}: n=2$ unable to complete post-physiotherapy LCl (leaks); ${ }^{+}: n=4$ unable to complete post-physiotherapy measurements final day ( $n=2$ discharged, $n=1 \mathrm{LCl}$ error, $\mathrm{n}=1 \mathrm{LCl}$ leaks). 
Patients used their usual airway clearance technique and this was repeated until there were two clear cycles producing no sputum. After a rest of $60 \mathrm{~min}$, LCI and PFT were repeated. Patients had a HRCT prior to assessments on one of their visits (unless $<6$ months HRCT available).

Exacerbating participants completed the same assessments as the stable patients except lung volumes to minimise fatigue. These data were collected twice: visit 1 within $48 \mathrm{~h}$ of commencing intravenous antibiotics and visit 2, on reaching clinical recovery at discharge as determined by the consultant. Patients underwent a HRCT within the first $48 \mathrm{~h}$ of admission.

Healthy controls were recruited from staff and family of the patients. Current smokers were excluded. They completed LCI and PFTs in triplicate on a single occasion.

\section{Measurements \\ Lung clearance index}

Innocor (Innovision A/S, Odense, Denmark) was used for performing LCI modified to include a separate Pneumotach (Hans Rudolph, Shawnee, KS), using 0.2\% sulfur hexafluoride (SF6) as the tracer gas (BOC, Guildford, UK) in a bias-flow system as previously reported [10] (online supplement).

\section{Pulmonary function tests}

These were completed in accordance with standardised criteria [18]. Flow/volumes were measured using a spirometer (Jaeger Master Screen PFT: Carefusion UK Ltd, Basingstoke, UK), lung volumes using constant body plethysmography ( Jaeger Master Screen Body; Carefusion UK Ltd). Transfer capacity of the lung for carbon monoxide (TLCO) with a single breath technique adjusted for alveolar volumes (KCO) (Jaeger Master Screen PFT Equipment; Carefusion UK Ltd); measurements corrected for haemoglobin [19] using a haemoglobinometer (Hemocue Hb 201 DM; Prospect Diagnostics Ltd, Dronfield, UK). Effective alveolar volume (VA) from TLCO measurement was expressed as a percent of total lung capacity (TLC) [20].

HRCT scoring

HRCTs were scored by two independent chest radiologists as previously described [21] (see online supplement).

\section{Statistical methods}

SPSSv22 (IBM Corp., Armonk, NY, USA) was used. Data are given as mean and standard deviation (SD), unless otherwise described. PFTs were presented as data or z-scores (standard residual). Significance levels were $\mathrm{p}<0.05$. One-way ANOVA tests with Bonferroni corrections and paired t-tests compared sets of normally distributed data. Coefficient of variation of LCI measurements was calculated as: (SD of two or three measurements/mean of three measurements) $\times 100$. Correlations were assessed with Spearman's rho for non-parametric data [22]. Reproducibility was evaluated with intra-class correlations coefficients and expressed as a proportion with the coefficient of variation. Bland-Altman plots were also performed to further analyse variability of LCI.

For the sample number calculation we used a standard deviation of the mean difference of 1.8 [11] and calculated a requirement of 24 patients in each group to detect a difference of 1.1 [15] in the LCI score with a power of $80 \%$ at a significance level of $<0.05$. Consequently we aimed to recruit 32 patients in each group allowing for dropouts.

\section{Results}

90 participants were recruited; 26 healthy controls, 32 stable bronchiectasis patients and 32 exacerbating bronchiectasis patients. One healthy control was excluded because of abnormal FEV1 z-score $(<-1.645)$ [23], three "stable" patients were unwell on the day of assessment and excluded and four exacerbating patients were unable to perform LCI measurements, for technical reasons. Four out of 29 stable patients did not attend visit 2 and three out of 28 exacerbating patients were discharged before the end of exacerbation assessment. Some data were incomplete as a few patients were unable to complete both preand post-physiotherapy data due to leaks/technical issues (figure 1a and b).

The demographics of the patients are detailed in table 1. There was no significant difference in the age, sex, aetiology or smoking history between the groups.

The LCI measurements were significantly lower $(\mathrm{p}<0.001)$ in the healthy control $(7.36 \pm 0.99)$ compared with the visit 1 (pre-physiotherapy) of the stable bronchiectasis (11.92 \pm 3.39 ) and day one (pre-physiotherapy) of the exacerbation bronchiectasis $(12.76 \pm 3.47)$ groups. There was no significant difference between the bronchiectasis groups. There was a moderate correlation between LCI and age in the healthy controls $(\mathrm{R}=0.590, \mathrm{p}=0.002)$ with an approximate increase of LCI of 0.4 per decade (figure 2$)$. 
TABLE 1 Demographics, pulmonary function tests, high-resolution computed tomography and lung clearance index (LCI) measurements for all groups

\begin{tabular}{|c|c|c|c|c|}
\hline & $\begin{array}{l}\text { Healthy } \\
\text { control }\end{array}$ & $\begin{array}{c}\text { Stable } \\
\text { bronchiectasis }\end{array}$ & $\begin{array}{c}\text { Exacerbation } \\
\text { bronchiectasis }\end{array}$ & p-value \\
\hline Subjects & 25 & 29 & 28 & \\
\hline Sex M/F & $11 / 14$ & $11 / 16$ & $11 / 18$ & All comparisons: NS \\
\hline Pseudomonas presence $n / N$ total & & $19 / 29$ & $21 / 27$ & All comparisons: NS \\
\hline \multicolumn{5}{|l|}{ Smoking history } \\
\hline$>10$ years & 1 & 2 & 3 & All comparisons: NS \\
\hline$>5$ years & 4 & 6 & 3 & All comparisons: NS \\
\hline Never & 20 & 21 & 22 & All comparisons: NS \\
\hline Oral steroid use & & 4 & 5 & All comparisons: NS \\
\hline $\mathrm{LCl}$ & $7.36 \pm 0.99$ & $11.91 \pm 3.39$ & $12.76 \pm 3.47$ & $\begin{array}{l}\text { HC versus } s B x: p<0.01 \\
\text { HC versus } \text { eBx: } p<0.01 \\
\text { sBx versus } \text { eBx: NS }\end{array}$ \\
\hline FRC z-score & $0.43 \pm 0.80$ & $1.18 \pm 1.54$ & $\mathrm{n} / \mathrm{a}$ & HC versus sBx: $p<0.01$ \\
\hline TLC z-score & $1.31 \pm 0.57$ & $0.82 \pm 1.75$ & $\mathrm{n} / \mathrm{a}$ & $\mathrm{HC}$ versus $\mathrm{sBx}: \mathrm{p}<0.05$ \\
\hline RV z-score & $0.21 \pm 0.71$ & $2.07 \pm 2.48$ & $\mathrm{n} / \mathrm{a}$ & HC versus sBx: $p<0.01$ \\
\hline RV/TLC z-score & $-0.79 \pm 0.80$ & $1.41 \pm 0.88$ & $\mathrm{n} / \mathrm{a}$ & HC versus $s B x: p<0.01$ \\
\hline TLco z-score & $-0.03 \pm 1.15$ & $-1.62 \pm 3.36$ & $\mathrm{n} / \mathrm{a}$ & HC versus $s B x: p<0.01$ \\
\hline$V_{A} / T L C$ & $0.88 \pm 0.35$ & $0.71 \pm 0.11$ & $\mathrm{n} / \mathrm{a}$ & HC versus $s B x: p<0.01$ \\
\hline Kco z-score & $-0.73 \pm 1.00$ & $-0.72 \pm 1.18$ & $\mathrm{n} / \mathrm{a}$ & All comparisons: NS \\
\hline Severity (max score of 4) & & $1.48(0.17-3.91)$ & $1.79(0.5-3.50)$ & All comparisons: NS \\
\hline
\end{tabular}

Data are presented as $\mathrm{n}$, mean \pm SD or median (range), unless otherwise stated. Comparisons were performed using ANOVA for more than two groups and t-test for two groups. Median differences were compared with Mann-Whitney U-test. Smoking history was defined as number of years smoking. Stable bronchiectasis: visit 1 stable bronchiectasis; exacerbation bronchiectasis: day 1-3 of intravenous antibiotics admission lall scores pre physiotherapy). M: Male; F: Female; FEV1: forced expiratory volume in $1 \mathrm{~s}$; FVC: forced vital capacity; FRC: functional residual capacity; TLC: total lung capacity; RV: residual volume; TLCO: transfer capacity of the lung for CO; $V_{A}$ : alveolar volume; Kco: transfer coefficient of the lung for CO; PI: post-infective; ABPA: allergic bronchopulmonary aspergillus; UC: ulcerative colitis; PCD: primary ciliary dyskinesia; CVID: chronic variable immune deficiency; HC: healthy control; sBx: stable bronchiectasis; eBx: exacerbation bronchiectasis; Ns: not significant.

\section{Reproducibility of $\mathrm{LCl}$}

The coefficient of variation of the three LCI measurements on one occasion was $4.1 \%$ in healthy controls, $4.5 \%$ in stable bronchiectasis and $5.0 \%$ during exacerbation. Inter-visit reliability within the stable group was also assessed with intraclass correlations coefficients between the LCI scores of the stable patients between first and second visits. The intraclass correlations coefficients for the between visit 1 and visit 2 variability (pre-physiotherapy) was 0.978 (95\% CI: 0.948-0.991), $\mathrm{p}<0.001(\mathrm{n}=25)$. A Bland-Altman plot comparing visit 1 and visit 2 LCI (pre-physiotherapy) (figure 3) demonstrated minimal variability between visits. This suggested that changes larger than 1.16 units (mean of difference between visits \pm 2 SDS or $\pm 95 \%$ CI) are outside normal variability.

\section{Relationship between LCl and PFTs}

The healthy controls had an LCI of $7.36 \pm 0.99$ and all had an FEV1 z-score above the lower limit of normal $(>-1.645) .12(41 \%)$ out of 29 patients in the stable group had a normal FEV1 $\mathrm{z}$-score; however, eight of 
FIGURE 2 Scatter plot of lung clearance index (LCl) scores and age in the healthy control group.

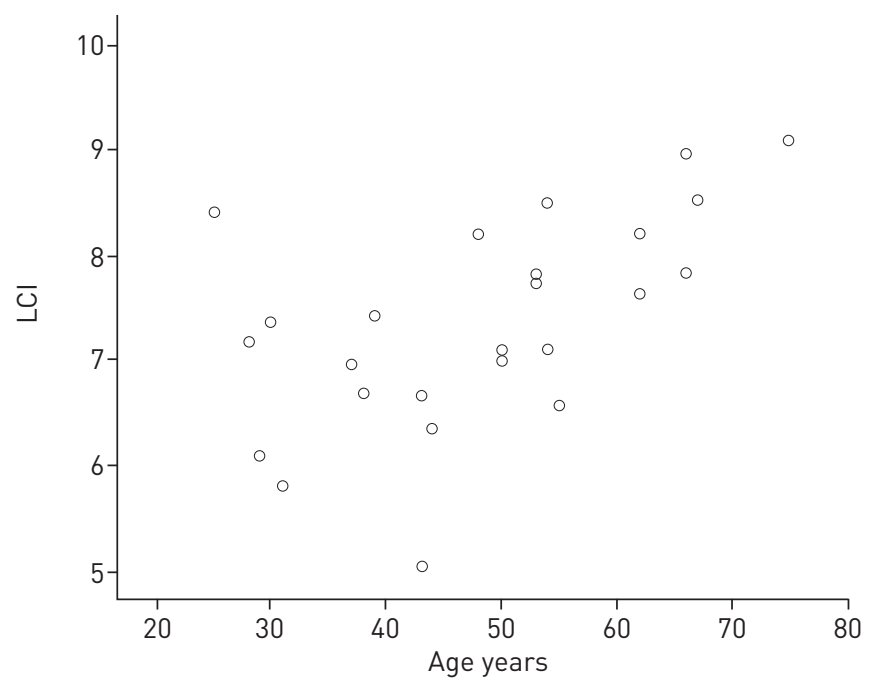

these had an abnormal LCI (>1.96 sD healthy control LCI (9.30)). In the exacerbating group 5 (18\%) out of 28 had normal FEV1 z-scores, of which two had an abnormal LCI. LCI measurements were strongly correlated with PFTs in stable bronchiectasis and exacerbation bronchiectasis (table 2). In addition to spirometric measures, there were strong correlations between LCI and PFTs measures of volume, gas trapping and small airways obstruction. There were no correlations in the healthy control group.

\section{Responsiveness of $\mathrm{LCl}$ to physiotherapy}

In the stable patients (table 3), there was no significant difference in LCI scores pre- and post-physiotherapy with a mean $\pm \mathrm{SD}$ difference of $0.26 \pm 0.99, \mathrm{p}=0.218$. However, there were statistically significant improvements in FEV1 z-score of $0.09 \pm 0.15 \mathrm{p}=0.006$, but these changes were clinically small with a mean change of $0.11 \pm 0.23 \mathrm{~L}$. There were no significant changes in FVC, RV, RVTLC and TLCO z-scores (data not shown). In exacerbation patients, LCI was not responsive to physiotherapy at either the beginning or end of an admission. As in stable patients there were some statistically significant improvements in FEV1 $\mathrm{z}$-scores following physiotherapy. The largest improvements, $0.13 \pm 0.25(\mathrm{p}=0.012)$, were at the start of an exacerbation, however, as in the stable state, changes were small with a mean change of $0.18 \pm 0.54 \mathrm{~L}$ at the start and $0.11 \pm 0.39 \mathrm{~L}$ at the end (table 3 ). There were no significant changes in FVC z-scores (data not shown).

\section{Responsiveness of $\mathrm{LCl}$ to treatment of an infective exacerbation}

There was no significant change in LCI or FEV1 z-score between the initial and last day (clinical recovery). The improvement in absolute FEV1 was $0.07 \pm 0.21 \mathrm{~L}$. There was a small but significant improvement in $V A$ of $0.17 \mathrm{~L} \mathrm{p}=0.032$ (table 4 and figure 4). There were no significant changes in FVC z-score (data not shown).

FIGURE 3 Bland-Altman plot of the difference between lung clearance index (LCI) measured on two separate visits and a mean of the two measurements.

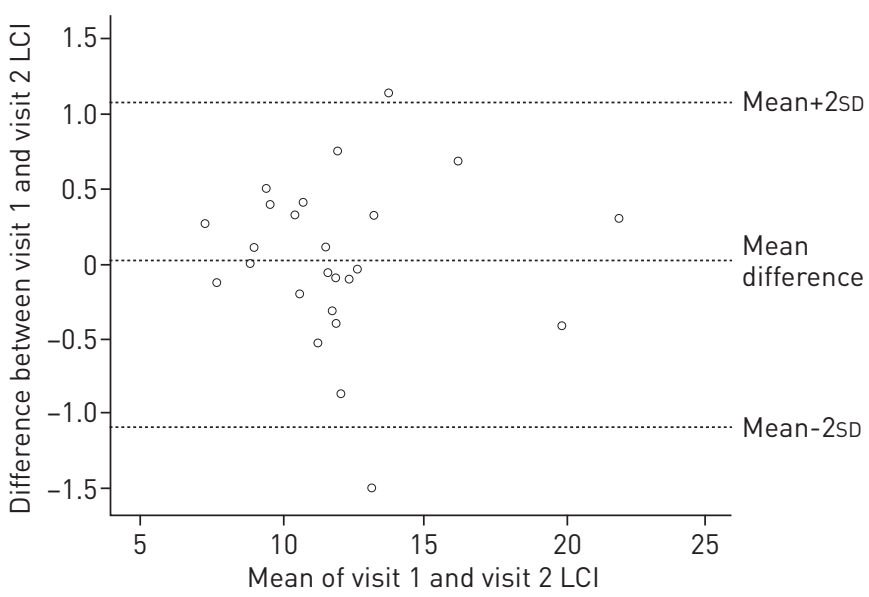


TABLE 2 Correlation of lung clearance index (LCI) with measurements of pulmonary function tests (PFTs) in all groups

\begin{tabular}{|c|c|c|c|}
\hline PFTs & LCI healthy controls & LCl stable & LCl exacerbation \\
\hline Subjects n & 25 & 29 & 28 \\
\hline FEV1 z-score & $0.206^{\#}$ & $-0.672^{*}$ & $-0.548 *$ \\
\hline FVC z-score & $0.184^{\#}$ & $-0.502^{\text {I }}$ & $-0.502^{\text {ๆ }}$ \\
\hline TLC z-score & $-0.571^{\#}$ & $0.341^{\#}$ & $\mathrm{n} / \mathrm{a}$ \\
\hline RV z-score & $-0.393^{\#}$ & $0.663^{\text {I }}$ & $\mathrm{n} / \mathrm{a}$ \\
\hline RV/TLC z-score & $-0.036^{\#}$ & $0.736^{\text {ๆ }}$ & $\mathrm{n} / \mathrm{a}$ \\
\hline FRC z-score & $0.286^{\#}$ & $0.596^{\text {ๆ }}$ & $\mathrm{n} / \mathrm{a}$ \\
\hline TLco Z-score & $-0.571^{\#}$ & $-0.403^{*}$ & $-0.641^{\text {ๆ }}$ \\
\hline$V_{A}$ & $-0.236^{\#}$ & $-0.444^{*}$ & $-0.584^{*}$ \\
\hline Kco z-score & $-0.014^{\#}$ & $-0.109^{\#}$ & -0.048 \\
\hline$V_{A} / T L C$ & $0.089^{\#}$ & $-0.787^{\Uparrow}$ & $\mathrm{n} / \mathrm{a}$ \\
\hline
\end{tabular}

Data are presented as results of a Spearman's test of correlation, unless otherwise stated. FEV 1 : forced expiratory volume in $1 \mathrm{~s}$; FVC: forced vital capacity; TLC: total lung capacity; RV: residual volume; FRC: functional residual capacity; $T \mathrm{LCO}$ : transfer capacity of the lung for $\mathrm{CO}$; $V_{\mathrm{A}}$ : alveolar volume; $K \mathrm{CO}$ : transfer coefficient of the lung for $\mathrm{CO} .{ }^{*}$ : nonsignificant; *: $p<0.05 ;{ }^{\text {ๆl: }} \mathrm{p}<0.01$.

\section{Discussion}

Here we report on the relative sensitivity of LCI and standard pulmonary function tests to two treatments of bronchiectasis, namely airway clearance and intravenous antibiotics. Contrary to our hypothesis, LCI did not change with treatment, but the better sensitivity of standard lung function tests was shown by statistically significant, albeit clinically very small, changes in lung function tests.

A previous study has demonstrated feasibility of LCI in stable bronchiectasis patients [11]. However this is the first study in an exacerbation. We show that the measure remains feasible in this group although the test did take longer to complete in the more severe and exacerbating patients. Moreover, three patients in the exacerbation group were unable to complete the measurement due to being unable to tolerate breathing through the mouthpiece. These patients had FEV1 z-score $<-4.0$ (mean FEV1 of $0.86 \mathrm{~L}$ ) and lower resting oxygenation. Previous studies have described how this measurement is more difficult to complete in more severe disease [11].

Within visit reproducibility is similar to previous studies $[8,10,11]$ and we show for the first time that this remains the case in during exacerbations. In stable bronchiectasis, LCI is also reproducible between visits at least 1 week apart. We estimated that a difference in LCI of 1.16 is a statistically significant change outside the expected variance; whether a change of this magnitude is clinically important awaits further study.

TABLE 3 Table to show impact of physiotherapy on lung clearance index (LCI) and other markers

Difference
pre/post-physiotherapy

\begin{tabular}{lrcc}
\hline Stable bronchiectasis physiotherapy $(\mathbf{n}=\mathbf{2 5})$ & & & \\
LCI & $0.26 \pm 0.99$ & 0.218 & $-0.93,0.17$ \\
FEV1 z-score & $-0.09 \pm 0.15$ & $0.006^{*}$ & $-0.15,-0.026$ \\
VA & $-0.07 \pm 0.14$ & $0.021^{*}$ & $-0.13,-0.01$ \\
VA/TLC & $-0.015 \pm 0.12$ & 0.502 & $-0.03,0.06$ \\
Start exacerbation physiotherapy (n=28) & & & \\
LCl & $0.11 \pm 0.85$ & 0.505 & $-0.44,0.22$ \\
FEV 1 z-score & $-0.13 \pm 0.25$ & $0.012 *$ & $-0.23,-0.03$ \\
VA & $-0.76 \pm 0.21$ & 0.064 & $-0.16,0.01$ \\
End exacerbation physiotherapy (n=21) & & & \\
LCl & $0.14 \pm 0.93$ & 0.491 & $-0.57,0.29$ \\
FEV1 z-score & $-0.08 \pm 0.12$ & $0.037 *$ & $-0.16,-0.01$ \\
VA & $-0.03 \pm 0.28$ & 0.498 & $-0.12,0.06$ \\
\hline
\end{tabular}

Data are presented as mean $\pm S D$, unless otherwise stated. Results displayed as mean differences with t-tests. FEV1: forced expiratory volume in $1 \mathrm{~s}$; VA: alveolar volume; TLC: total lung capacity. ${ }^{*}$ : $p<0.05$. 
TABLE 4 Table to show impact of clinical improvement from exacerbation on lung clearance index (LCI) in exacerbation patients

Start versus end exacerbation bronchiectasis $(n=25)$

\begin{tabular}{lccc} 
& Difference initial-last day & $\mathbf{p}$-value & $\mathbf{9 5 \%} \mathbf{C l}$ \\
\hline LCI & $0.38 \pm 1.59$ & 0.234 & $-0.15,0.95$ \\
FEV 1 z-score & $-0.08 \pm 0.25$ & 0.285 & $0.22,-0.07$ \\
VA & $-0.17 \pm 0.39$ & $0.032 *$ & $-0.32,-0.02$ \\
\hline
\end{tabular}

Datra are presented as mean $\pm \mathrm{SD}$, unless otherwise stated. $\mathrm{FEV} 1$ : forced expiratory volume in $1 \mathrm{~s}$; $V_{\mathrm{A}}$ : alveolar volume

The healthy controls were age-matched (mean age of 49.9 years) whereas previous studies [10, 11] have been predominantly in children or younger adults. The mean LCI of healthy controls in this study was $7.36 \pm 0.99$, suggesting an upper limit of normal (ULN) of 9.3 (1.96 SD >mean) [10]. This is higher than previously published data where the ULN has been reported as 7.5 [10]. The modest correlation between LCI and age in this population is different to previous data in bronchiectasis [10] where no correlation was found but consistent with nitrogen multiple breath washout data, which suggested a small effect $(0.22$ per decade) of age on LCI [24]. This apparent age related effect in the data must be read together with the knowledge that the sampling of our older age groups is not as good as for younger subjects (figure 2) and consequently further data is required to confirm this effect.

This study is the first to compare LCI with full PFTs (including spirometry, lung volumes and gas transfer). As in previous studies [11,25], there is a significant correlation between LCI and FEV1 in the bronchiectasis cohort. This is in contrast with results demonstrated in a study of primary ciliary dyskinesia (PCD) patients $[26,27]$ although in this study, many had a normal FEV1, unlike our population. There were two PCD patients in this study, who were recruited before the potential different relationship had been reported. These patients were retained in the study as per the study design. Additionally, removal of these patients from the analysis did not alter the results.

In addition to spirometric markers, this study demonstrated significant correlations with measures of air trapping in the stable group. Moreover the stable bronchiectasis group also showed a significant correlation with VA/TLC, a simple measure of gas mixing [16]. Few studies have looked at these relationships with LCI. A study in paediatric CF [28] concurred with these results, demonstrating the strongest correlations of LCI with measures of hyperinflation including RV, RV/TLC and FRC rather than spirometric measures. Furthermore, evidence from HRCT in bronchiectasis suggests scores of gas trapping are associated with small airways disease, evidenced from mosaic attenuation on expiratory HRCT [29], which supports the relationships seen between LCI and lung volumes.

LCI is not responsive immediately post physiotherapy as an intervention in either the stable or the exacerbating state. Other studies have assessed the response of a variety of indices to short-term physiotherapy in stable patients with CF. One study used helium magnetic resonance imaging pre- and post-airway clearance to assess the short-term changes of physiotherapy [30]. This demonstrated that although there was a change in ventilation there was no overall improvement in ventilation inhomogeneity.
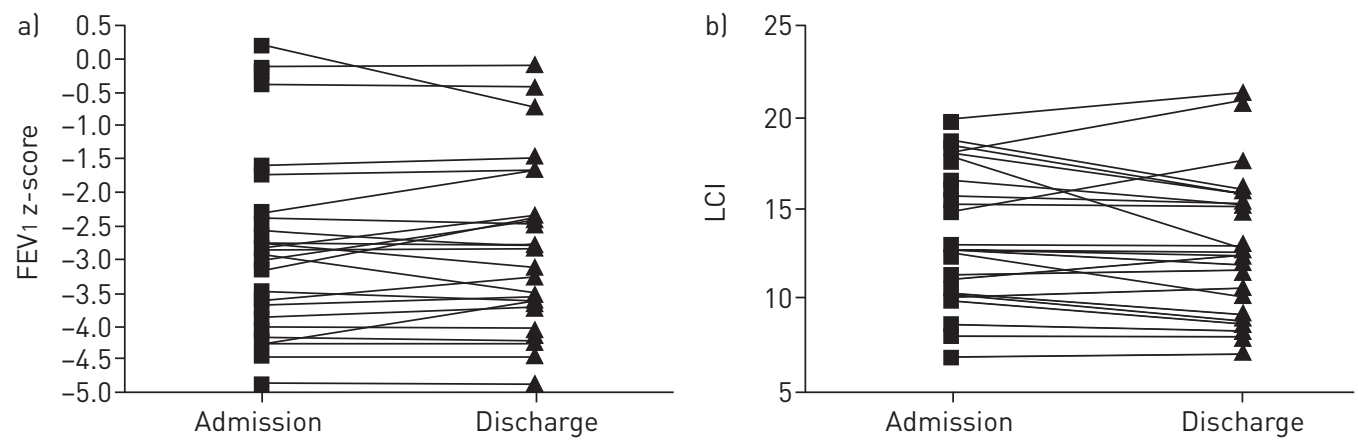

FIGURE 4 a) Forced expiratory volume in $1 \mathrm{~s}\left(\mathrm{FEV}_{1}\right)$ z-scores at the beginning of admission compared to the end of the admission; and b) lung clearance index (LCI) scores at the beginning of admission compared to the end of the admission. 
Additionally, short-term physiotherapy was not found to have a consistent effect on the LCI results in a cohort of stable CF patients [17]. Our study demonstrated similar results with LCI in response to physiotherapy in stable patients. We also investigated the impact of physiotherapy in acutely exacerbating patients and the same clinically recovered patients where more measurable impact may be expected.

There was also no change in LCI following inpatient intravenous antibiotic therapy for an infective exacerbation. This intervention provided significant clinical improvement and so may have been expected to produce a significant improvement in LCI. One explanation for the failure of the response of LCI in this setting is the nature of the improvements on the background of significant underlying lung disease. LCI is insensitive to an occluded airway, and airways with very long time constants, and the measurements do not take into account the severity of the lung disease distally. Significant clinical improvements may follow a reduction in mucus plugging, however in some cases this could lead to a paradoxical worsening in LCI as the distal areas of more diseased lung contribute to the LCI score. Alternatively, if there is improvement in gas mixing in other areas of the lung, the net result could be no change. Supporting this hypothesis is the improvement in VA in the exacerbating patients whose LCI worsened. Similar hypotheses could also be used to explain the lack of response to physiotherapy during an acute exacerbation in this study and have also been forwarded to explain the inconsistent results of LCI at tracking a recovery from an exacerbation in CF [14]. Unlike in CF, where FEV1 can be used as a positive control for improvement, there are no good objective markers for improvement following treatment for an exacerbation in bronchiectasis. Consistent with this, FEV1 z-score did not significantly improve in this study, with some variability of response (table 4 and figure 4). This helps stress the importance of marker discovery in bronchiectasis but does also propose an alternative explanation in this cohort for the insensitivity of LCI to exacerbation treatment. It is possible that this intervention was unsuccessful and that there was no significant clinical improvement to be measured by LCI. This is however not likely in this study. All patients reached clinical recovery at the end of the course of intravenous antibiotics, including improvements in patient's symptoms and sputum production. Furthermore, although small, there were improvements in VA following treatment suggestive of clinical improvement, and showing that LCI is a less sensitive physiological marker, contrary to our hypothesis.

Possible limitations of the study include the fact that it has been performed in a tertiary centre and the result may therefore not be directly applicable to all bronchiectasis cohorts. In particular, the exacerbating patients were a severely ill population (mean FEV1 $\mathrm{z}$-score -2.97) and improvements in these markers may take a longer time. Additionally, LCI has previously been shown to have increased regional variation as airflow obstruction increases [31]. In this study, it is also difficult to make direct comparisons between the stable and exacerbating bronchiectasis patients, as they were different cohorts.

The study does however have significant strengths. It is a prospective study that reports for the first time the (lack of) responsiveness of a new potential marker for bronchiectasis. In addition, it uses contemporaneous full lung function testing, and age-matched healthy controls. Moreover evaluation of airway clearance has not previously been completed in this acutely exacerbating population before.

Although LCI was not a useful marker to short-term physiotherapy or recovery from an exacerbation, it may still be useful as a marker and endpoint in other circumstances. LCI may also be more responsive in less severe patients. Furthermore, whereas it may be insensitive to short-term interventions that lead to predominantly mucus clearance it may be much more responsive to longer term treatments that may impact on other aspects of the pathology, such as airway inflammation or thickening [32]. Such improvements may improve ventilation throughout the lung and not produce the inconsistencies, which may result from the clearance of impacted airways. Future assessments of LCI and other clinical endpoints in long-term studies of anti-inflammatories or antibiotics in bronchiectasis are needed, as discovery of such markers is necessary to help improve management and the evidence base of this disease.

\section{References}

1 Pasteur MC, Bilton D, Hill AT, et al. British Thoracic Society guideline for non-CF bronchiectasis. Thorax 2010; 65: Suppl 1, i1-i58.

2 Bilton D, Loebinger MR, Wilson R. Non-cystic fibrosis bronchiectasis: an evidence-base for new therapies. Lancet Respir Med 2014; 2: 958-960.

3 Chalmers JD, Smith MP, McHugh BJ, et al. Short- and long-term antibiotic treatment reduces airway and systemic inflammation in non-cystic fibrosis bronchiectasis. Am J Respir Crit Care Med 2012; 186: 657-665.

4 Smith MP, Hill AT. Evaluating success of therapy for bronchiectasis: what end points to use? Clin Chest Med 2012; 33: 329-349.

5 Chalmers JD. Bronchiectasis trials: losing the battle but winning the war? Lancet Respir Med 2014; 2: 679-681.

6 Davies JC, Cunningham S, Alton EW, et al. Lung clearance index in CF: a sensitive marker of lung disease severity. Thorax 2008; 63: 96-97.

7 Fuchs SI, Gappa M. Lung clearance index: clinical and research applications in children. Paediatr Respir Rev 2011; 12: 264-270. 
Fuchs SI, Eder J, Ellemunter H, et al. Lung clearance index: normal values, repeatability, and reproducibility in healthy children and adolescents. Pediatr Pulmonol 2009; 44: 1180-1185.

9 Gustafsson PM, De Jong PA, Tiddens HA, et al. Multiple-breath inert gas washout and spirometry versus structural lung disease in cystic fibrosis. Thorax 2008; 63: 129-134.

10 Horsley AR, Gustafsson PM, Macleod KA, et al. Lung clearance index is a sensitive, repeatable and practical measure of airways disease in adults with cystic fibrosis. Thorax 2008; 63: 135-140.

11 Rowan SA, Bradley JM, Bradbury I, et al. Lung clearance index is a repeatable and sensitive indicator of radiological changes in bronchiectasis. Am J Respir Crit Care Med 2014; 189: 586-592.

12 Amin R, Subbarao P, Lou W, et al. The effect of dornase alfa on ventilation inhomogeneity in patients with cystic fibrosis. Eur Respir J 2011; 37: 806-812.

13 Amin R, Subbarao P, Jabar A, et al. Hypertonic saline improves the LCI in paediatric patients with CF with normal lung function. Thorax 2010; 65: 379-383.

14 Horsley AR, Davies JC, Gray RD, et al. Changes in physiological, functional and structural markers of cystic fibrosis lung disease with treatment of a pulmonary exacerbation. Thorax 2013; 68: 532-539.

15 Rosenfeld M, Ratjen F, Brumback L, et al. Inhaled hypertonic saline in infants and children younger than 6 years with cystic fibrosis: the ISIS randomized controlled trial. JAMA 2012; 307: 2269-2277.

16 Hughes JM, Pride MB. Examination of the carbon monoxide diffusing capacity (DL(CO)) in relation to its KCO and VA components. Am J Respir Crit Care Med 2012; 186: 132-139.

17 Davies J, Sheridan H, Bell N, et al. Assessment of clinical response to Ivacaftor with lung clearance index in cystic fibrosis patients with a G551D-CFTR mutation and preserved spirometry: a randomised controlled trial. Lancet Respir Med 2013; 1: 630-638.

18 Fuchs SI, Toussaint S, Edlhaimb B, et al. Short-Term Effect of Physiotherapy on Variability of the Lung Clearance Index in Children With Cystic Fibrosis. Paediatr Pulmonol 45: 301-306.

19 Hyatt RE, Scanlon PD, Nakamura MMD. Interpretation of pulmonary function tests: a practical guide. 3rd Edn. Philadelphia, Lippincott Williams \& Wilkins, 2009.

20 Marrades RM, Diaz O, Roca J, et al. Adjustment of DLCO for hemoglobin concentration. Am J Respir Crit Care Med 1997; 155: 236-241.

21 Loebinger MR, Wells AU, Hansell DM, et al. Mortality in bronchiectasis: a long-term study assessing the factors influencing survival. Eur Respir J 2009; 34: 843-849.

22 Munro BH. Statistical methods for health care research. 5th Edn. Philadelphia, Lippincott Williams \& Wilkins, 2005.

23 Stanojevic S, Wade A, Stocks J. Reference values for lung function: past, present and future. Eur Respir J 2010; 36: $12-19$.

24 Verbanck S, Thompson BR, Schuermans D, et al. Ventilation heterogeneity in the acinar and conductive zones of the normal ageing lung. Thorax 2012; 67: 789-795.

25 Gonem S, Scadding A, Soares M, et al. Lung clearance index in adults with non-cystic fibrosis bronchiectasis. Respir Res 2014; 15: 59.

26 Green K, Buchvald FF, Marthin JK, et al. Ventilation inhomogeneity in children with primary ciliary dyskinesia. Thorax 2012; 67: 49-53.

27 Irving SJ, Ives A, Davies G, et al. Lung clearance index and high-resolution computed tomography scores in primary ciliary dyskinesia. Am J Respir Crit Care Med 2013; 188: 545-549.

28 Owens CM, Aurora P, Stanojevic S, et al. Lung Clearance Index and HRCT are complementary markers of lung abnormalities in young children with CF. Thorax 2011; 66: 481-488.

29 Roberts HR, Wells AU, Milne DG, et al. Airflow obstruction in bronchiectasis: correlation between computed tomography features and pulmonary function tests. Thorax 2000; 55: 198-204.

30 Woodhouse N, Wild JM, van Beek E, et al. Assessment of hyperpolarized 3He lung MRI for regional evaluation of interventional therapy: a pilot study in pediatric cystic fibrosis. J Magn Reson Imaging 2009; 30: 981-988.

31 Robinson PD, Cooper P, Van Asperen P, et al. Using index of ventilation to assess response to treatment for acute pulmonary exacerbation in children with cystic fibrosis. Pediatr Pulmonol 2009; 44: 733-742.

32 Kraemer R, Latzin P, Pramana I, et al. Long-term gas exchange characteristics as markers of deterioration in patients with cystic fibrosis. Respir Res 2009; 10: 106. 